\title{
Making the Perfect Winter Wonderland-A Case Report of Infectious Crystalline Keratopathy in the Setting of Chronic Nepafenac Use for Cystoid Macular Edema
}

Luke Y Chang, ${ }^{1}$ Justin Hess, ${ }^{1,2}$ Zachary Lukowski, ${ }^{1}$ Amy J Estes ${ }^{1}$

1. Department of Ophthalmology, Medical College of Georgia, Augusta University, Augusta, GA, USA; 2. Brody School of Medicine, East Carolina University, Greenville, NC, USA

DOI: https://doi.org/10.17925/USOR.2019.12.2.96

\begin{abstract}
A n 80-year-old woman with cystoid macular edema on concurrent nepafenac and prednisolone therapy presented for routine followup with no complaints. However, on exam, she was found to have multiple feathery-bordered corneal infiltrates and a near complete epithelial defect. Corneal cultures grew pan-sensitive Streptococcus mitis. The patient required prolonged fortified antibiotic therapy before resolution of infiltrates and epithelial defect. The patient's presentation and clinical course were most consistent with infectious crystalline keratopathy (ICK). Although ICK is classically associated with an intact corneal epithelium, such as after penetrating keratoplasty, this patient developed a near-complete epithelial defect from chronic nepafenac use and localized immunosuppression by prednisolone led to the development of ICK. This is an unusual presentation of ICK in the setting of prolonged topical nonsteroidal anti-inflammatory drug (NSAID) use. This case raises awareness for a rare potential complication of chronic topical NSAID use.
\end{abstract}

\section{Keywords}

Infectious crystalline keratopathy, nepafenac, chronic NSAID use, cystoid macular edema

Disclosures: Luke Chang, Justin Hess, Zachary Lukowski, and Amy J Estes have no conflicts of interest to disclose. No funding was received in the publication of this article.

Acknowledgements: The authors would like to extend a special thanks to Mrs Sarah Tau and Mr Mike Stanley for their ophthalmic imaging expertise.

Review Process: Double-blind peer review.

Compliance with Ethics: All procedures were followed in accordance with the responsible committee on human experimentation and with the Helsinki Declaration of 1975 and subsequent revisions. Informed consent was received from the patients involved in the report.

Authorship: The named author meets the International Committee of Medical Journal Editors (ICMJE) criteria for authorship of this manuscript, takes responsibility for the integrity of the work as a whole, and has given final approval for the version to be published.

Received: August 28, 2019

Accepted: November 2, 2019

Citation: US Ophthalmic Review. 2019;12(2):96-7

Corresponding Author: Amy J Estes, Department of Ophthalmology, Augusta University, 1120 15th Street, BA-2720, Augusta, GA 30912. E: aestes@augusta.edu

Support: No funding was received in

the publication of this article.
Infectious crystalline keratopathy (ICK) is an uncommon and indolent infection classically characterized by densely packed branching opacities in the absence of a robust inflammatory response. This infection is most often seen in the setting of prior corneal surgery, particularly penetrating keratoplasty. ${ }^{1}$ The risk for developing ICK in these cases is potentiated by chronic localized immunosuppression to prevent graft rejection postoperatively.' In addition to local immunosuppression, the minimal inflammation in ICK has been attributed to the ability of causative organisms to produce a biofilm that can prevent inflammatory cell infiltration. ${ }^{12}$ Although many organisms have been implicated in ICK, Streptococcus viridans has been most commonly isolated. ${ }^{1-3}$

In this case, an 80-year-old woman developed ICK after concurrent topical nonsteroidal antiinflammatory (NSAID) and corticosteroid use for cystoid macular edema (CME). This is an unusual presentation of ICK reported in the setting of prolonged topical NSAID use in an eye without prior corneal surgery.

\section{Case report}

An 80-year-old Caucasian female presented for routine follow-up of CME in both eyes. Her ocular history was significant for primary open-angle glaucoma in both eyes, status post selective laser trabeculoplasty in the left eye, as well as bilateral cataract extraction. Her medical history was notable for hypertension, hyperthyroidism, and type 2 diabetes mellitus without complications. Her CME was diagnosed by optical coherence tomography (OCT) 10 weeks prior to presentation and was confirmed by intravenous fluorescein angiography revealing a petaloid leakage pattern in both eyes. She was treated with $1 \%$ prednisolone acetate and $0.1 \%$ nepafenac four times a day in both eyes.

At presentation, she had no complaints and stated that her vision was at baseline. OCT demonstrated resolution of CME in both eyes; however, her visual acuity had declined from 20/50 in each eye to 20/200 and 20/70 in the right and left eye, respectively. Slit lamp exam of her right eye showed multiple corneal infiltrates with feathery borders and a near complete epithelial defect (Figure 1) without cell or hypopyon. Her left eye had a large central epithelial defect without infiltrate. Cultures of the infiltrate were performed, nepafenac and prednisolone were discontinued, and fortified antibiotics (vancomycin $25 \mathrm{mg} / \mathrm{mL}$ and tobramycin $14 \mathrm{mg} / \mathrm{mL}$ ) and antifungals (amphotericin $1.5 \mathrm{mg} / \mathrm{mL}$ ) were started empirically. The left eye was treated with lubrication and moxifloxacin $(0.5 \%)$ for prophylaxis, 
Figure 1: Slit lamp photography at presentation

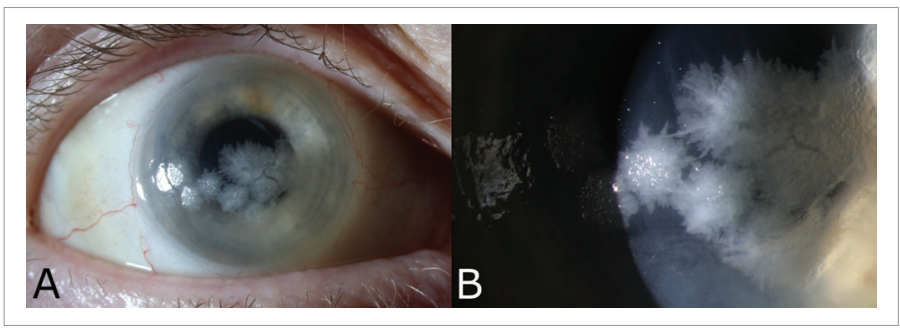

A. Slit lamp photograph demonstrating multiple infiltrates with feathery borders within a near complete epithelial defect. B. High magnification slit lamp photograph revealing classic "snowflake" appearance of infectious crystalline keratopathy.

and the epithelial defect resolved without complication. Cultures of the right eye ultimately grew pan-sensitive Streptococcus mitis, at which time the antifungals and tobramycin were discontinued.

She was followed daily for the first week with interval improvement in her corneal infiltrate and stable epithelial defect (Figure 2). The corneal infiltrates resolved by 1 month and epithelial defect resolved by 5 weeks. Prednisolone acetate $1 \%$ was initiated at 6 weeks to treat diffuse keratic precipitates and significant stromal scarring. Antibiotic drops were stopped after 8 weeks of therapy.

Final acuity was $2 / 200$ in the right eye with residual right stromal scarring and no evidence of infiltrate recurrence; final acuity in the left eye was 20/30. OCT demonstrated no recurrence of CME in either eye.

\section{Discussion}

ICK has largely been reported in the context of an intact epithelium ${ }^{3}$ with proliferation of bacteria in the anterior stroma, which contributes to the crystalline appearance., In addition to previous corneal surgery, other predisposing risk factors for ICK include epithelial disruption from laser in situ keratomileusis (LASIK), contact lens wear, preexisting infectious keratitis, and topical anesthetic abuse.
Figure 2: Slit lamp photography at 1 week follow-up

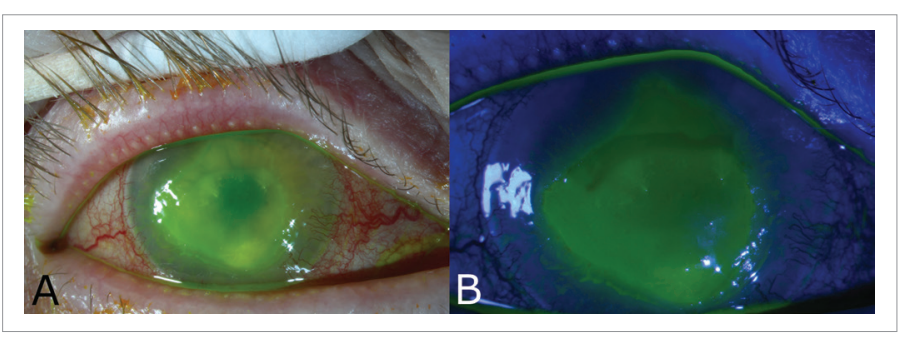

A. Slit lamp photography showing interval improvement in right eye corneal infiltrates with one dense infiltrate remaining inferiorly and small scattered foci at the periphery of the remaining epithelial defect which had not changed in size; also with evidence of new corneal neovascularization. B. Fluorescein staining demonstrating persistent epithelial defect measuring 5-mm vertically by 6-mm horizontally.

Topical NSAIDs have known corneal complications, ranging from superficial keratopathy to persistent epithelial defects, and even corneal melts or perforation. ${ }^{4-6}$ Topical NSAIDS have also been shown to delay epithelial wound healing. ${ }^{4-6}$ Corneal toxicity is mediated by NSAID inhibition of cox enzymes and subsequent production of prostaglandins, which are responsible for maintaining cellular integrity ${ }^{5}$ and mediating leukocyte migration. ${ }^{4}$ Nepafenac is a newer NSAID that is highly touted for its greater corneal penetration. ${ }^{5}$ Unfortunately, the manner in which increased corneal penetration affects therapeutic efficacy and corneal toxicity compared to other NSAIDS, remains unclear. However, nepafenac has been associated with delayed healing rates after flapless surface ablation when compared to ketorolac.

Our patient developed a large and persistent epithelial defect while on chronic nepafenac. In the context of her concurrent topical prednisolone therapy, localized immunosuppression created an environment favorable for the development of ICK. Although concomitant topical NSAID and topical corticosteroid may be a mainstay in the treatment of $\mathrm{CME}$, we propose that this combination be used with caution as prolonged exposure may predispose patients to infectious processes such as ICK. $\square$

1. Porter AJ, Lee GA, Jun AS. Infectious crystalline keratopathy. Surv Ophthalmol. 2018;63:480-99.

2. Georgiou T, Qureshi SH, Chakrabarty A, Noble BA. Biofilm formation and coccal organisms in infectious crystalline keratopathy. Eye. 2002;16:89-92.

Bowling B. Kanski's clinical ophthalmology: A systematic approach. Eighth edition. Edinburgh UK: Elsevier. 2016.

Kim SJ, Flach AJ, Jampol LM. Nonsteroidal anti-inflammatory drugs in ophthalmology. Surv Ophthalmol. 2010;55:108-33.

Gaynes BI, Onyekwuluje A. Topical ophthalmic NSAIDs: A discussion with focus on nepafenac ophthalmic suspension. Clin Ophthalmol. 2008;2:355-68.

6. Trattler W, McDonald M. Double-masked comparison of ketorolac tromethamine $0.4 \%$ versus nepafenac sodium $0.1 \%$ for postoperative healing rates and pain control in eyes undergoing surface ablation. Cornea. 2007;26:665-9. 